\title{
Synthesis, characterization, and antimicrobial properties of copper nanoparticles
}

This article was published in the following Dove Press journal:

International Journal of Nanomedicine

20 November 2013

Number of times this article has been viewed

\author{
Muhammad Sani Usman' \\ Mohamed Ezzat El \\ Zowalaty ${ }^{2,5}$ \\ Kamyar Shameli 1,3 \\ Norhazlin Zainuddin' \\ Mohamed Salama ${ }^{4}$ \\ Nor Azowa Ibrahim' \\ 'Department of Chemistry, Faculty \\ of Science, ${ }^{2}$ Laboratory of Vaccines \\ and Immunotherapeutics, Institute \\ of Bioscience, Universiti Putra \\ Malaysia, Selangor, Malaysia; ${ }^{3}$ Materials \\ and Energy, Research Center, Karaj, \\ Iran; ${ }^{4}$ Faculty of Pharmacy, UiTM, \\ Puncak Alam, Selangor, Malaysia; \\ ${ }^{5}$ Department of Environmental Health, \\ Faculty of Public Health and Tropical \\ Medicine, Jazan University, Jazan, \\ Kingdom of Saudi Arabia
}

\begin{abstract}
Copper nanoparticle synthesis has been gaining attention due to its availability. However, factors such as agglomeration and rapid oxidation have made it a difficult research area. In the present work, pure copper nanoparticles were prepared in the presence of a chitosan stabilizer through chemical means. The purity of the nanoparticles was authenticated using different characterization techniques, including ultraviolet visible spectroscopy, transmission electron microscopy, X-ray diffraction, Fourier transform infrared spectroscopy, and field emission scanning electron microscopy. The antibacterial as well as antifungal activity of the nanoparticles were investigated using several microorganisms of interest, including methicillin-resistant Staphylococcus aureus, Bacillus subtilis, Pseudomonas aeruginosa, Salmonella choleraesuis, and Candida albicans. The effect of a chitosan medium on growth of the microorganism was studied, and this was found to influence growth rate. The size of the copper nanoparticles obtained was in the range of 2-350 $\mathrm{nm}$, depending on the concentration of the chitosan stabilizer.
\end{abstract}

Keywords: chitosan, copper nanoparticles, antimicrobial activity, chemical synthesis, aqueous medium

\section{Introduction}

The high occurrence of resistance of different microorganisms to the majority of antimicrobial agents is attracting a great deal of attention. The World Organization for Animal Health, Food and Agriculture Organization, and World Health Organization have all commented on the serious threat posed by antimicrobial-resistant pathogenic organisms to human and animal health. ${ }^{1}$ The extensive use of chemotherapeutic antimicrobial agents has generated the selective pressure to encourage the escalating rates in antimicrobial resistance. ${ }^{2,3}$

Resistance of microorganisms to antibiotics is steadily rising, with reports showing that quite a number of the recognized antimicrobial agents in existence have demonstrated resistance by one species of microorganism or another, ${ }^{1}$ so basically there is no single antimicrobial agent available for human and animal use that has not demonstrated resistance by microorganisms. This development had compelled clinicians to rely on in vitro antimicrobial susceptibility testing for diagnostic purposes. ${ }^{4}$ In this regard, synthesis or extraction of compounds such as nanoparticles with antimicrobial properties is essential, and has potentially promising applications in the fight against the ever-growing number of antimicrobial-resistant pathogenic microorganisms which pose a continuous threat to human and animal health. 
Nanotechnology is a field of science with vast potential in medicine. Being analogous with nature, the combination of nanoscience and biology will not only strengthen the fight against pathogenic microorganisms but can also result in a change in approach towards combating infectious diseases. ${ }^{5}$ Consequently, diseases like cancer and rheumatoid arthritis are also being combated using nanoparticles ${ }^{6,7}$ Materials in the range of $100 \mathrm{~nm}$ or less are considered to be nanoparticles. They exhibit a wide range of properties, including optical, electrical, catalytic, ${ }^{8}$ magnetic, and biological activity, ${ }^{9}$ which is different from that of their bulk materials. Some of the biological properties of nanoparticles have been explored by antimicrobial susceptibility testing of nanoparticles produced from different metals using different synthetic methods. ${ }^{9}$ It has been reported that metal nanoparticles (Ag, $\mathrm{Cu}, \mathrm{CuO}, \mathrm{Au}$ ) exhibit a wide spectrum of antimicrobial activity against different species of microorganisms, including fungi and Gram-positive and Gram-negative bacteria. Antibacterial activity has been reported against Escherichia coli $^{10-12}$ and a nonresistant strain of Gram-positive bacteria (Staphylococcus aureus), ${ }^{11-13}$ and the results obtained indicate inhibition of growth of the organism due to addition of the nanoparticles.

Generally, metallic nanoparticles show antibacterial and antifungal activity, even though there are environmental and human safety concerns regarding the release and consumption of metal nanoparticles which are yet to be explored. Excessive release of silver, for example, causes environmental pollution which in turn makes silver harmful to humans and animals. Copper is no exception, because an excess of copper in the human body leads to generation of the most damaging radicals, such as the hydroxyl radical. ${ }^{14}$ However, there are copper-transporting adenosine triphosphatases (Cu-ATPases), including ATP7A and ATP7B, which play an important role in copper homeostasis and export excess copper through the intestine (ATP7A) as feces, the liver (ATP7B) as a bile product, and the mammary gland (ATP7B) as milk. ${ }^{14,15}$

The availability of copper has made it a better choice to work with, because it shares properties similar to those of other expensive noble metals, including silver and gold. The choice of copper in the present research is attributed to the above-mentioned factors; in addition, copper nanoparticles are reported to have antimicrobial activity against a number of species of bacteria and fungi. Previous studies have indicated that copper nanoparticles have antimicrobial activity against E. coli and Staphylococcus species, ${ }^{16,17}$ and similar antifungal properties were also reported. ${ }^{18}$
However, copper nanoparticles have major limitations, which include rapid oxidation on exposure to air. Copper oxidizes to $\mathrm{CuO}$ and $\mathrm{Cu}_{2} \mathrm{O}$, and converts to $\mathrm{Cu}^{2+}$ during preparation and storage, so it is difficult to synthesize copper nanoparticles in an ambient environment. Therefore, alternative pathways have been developed to synthesize metal nanoparticles in the presence of polymers (eg, polyvinylpyrrolidone, polyethylene glycol, and chitosan) and surfactants (cetyl trimethyl ammonium bromide) as stabilizers, and to form coatings on the surface of nanoparticles. Recently, plant extracts have been used to stabilize nanoparticles in green synthesis. ${ }^{19}$ In general, quite a number of nanoparticles are prepared using polymer dispersions. ${ }^{20-23}$ A number of techniques can be used to prepare copper nanoparticles, including thermal reduction, ${ }^{24}$ a capping agent method, ${ }^{25}$ sonochemical reduction, ${ }^{26}$ metal vapor synthesis, ${ }^{27}$ microemulsion techniques, ${ }^{28}$ laser irradiation, ${ }^{29}$ and induced radiation. ${ }^{30}$

The present study investigated the antimicrobial properties of metallic copper nanoparticles synthesized in chitosan polymer medium through chemical means. Chitosan is the second naturally occurring and most abundant biopolymer after cellulose, and is obtained by removal of an acetyl group from chitin. ${ }^{31}$ It is composed of glucosamine and $\mathrm{N}$-acetylglucosamine units. ${ }^{23}$ Chitosan is a biocompatible, biodegradable, and nontoxic polymer with various applications in the pharmaceutical and biomedical fields. ${ }^{31}$ These properties make the chitosan polymer a good candidate for medical applications and research. The antimicrobial activity of the copper nanoparticles synthesized in the present study was tested against various microorganisms of interest, including Gram-positive bacteria such as methicillin-resistant S. aureus (MRSA) and Bacillus subtilis, Gram-negative organisms such as Salmonella choleraesuis and Pseudomonas aeruginosa, and yeast species such as Candida albicans. The antimicrobial activity of copper nanoparticles against a number of microorganisms has been reported previously. ${ }^{32-36}$ However, it is interesting to note that our findings in this study indicate activity against all species tested. The ultimate purpose of this study was to analyze the effect of chitosan on the antimicrobial properties of copper nanoparticles synthesized by chemical means.

\section{Materials and methods Chemicals and preparation}

Analytical grade copper sulfate pentahydrate $\left(\mathrm{CuSO}_{4} \cdot 5 \mathrm{H}_{2} \mathrm{O}\right.$, 99.98\%) and ascorbic acid were supplied by Sigma-Aldrich (St Louis, MO, USA). Chitosan (molecular weight $600,000-800,000)$ and hydrazine $(98.0 \%)$ were supplied by 
Acros Organics (Thermo Fisher Scientific, Fair Lawn, NJ, USA), and sodium hydroxide (99.0\%) was sourced from R\&M Chemical Ltd (Edmonton, AB, Canada). Milli Q water (EMD Millipore Bedford, MA, USA) was used throughout the experiment. In the first step, $10 \mathrm{~mL}$ of $\mathrm{CuSO}_{4} \cdot 5 \mathrm{H}_{2} \mathrm{O}$ $(0.05 \mathrm{M})$ was added to $40 \mathrm{~mL}$ of acetic acid solution $(0.1 \mathrm{M})$ containing chitosan $(0.05,0.1,0.2$, and $0.5 \mathrm{wt} \%)$ to obtain a blue-colored solution. With constant stirring and refluxing at $120^{\circ} \mathrm{C}, 0.5 \mathrm{~mL}$ of ascorbic acid $(0.05 \mathrm{M})$ was added. In the second step, $2 \mathrm{~mL}$ of $\mathrm{NaOH}(0.6 \mathrm{M})$ was added to the solution after further stirring for 20 minutes, until a green solution was obtained. Finally, a $0.5 \mathrm{~mL}$ volume $(0.05 \mathrm{M})$ of $\mathrm{N}_{2} \mathrm{H}_{4}$ was added and deep red coloration was obtained, indicating formation of copper nanoparticles. The nanoparticles were isolated by centrifugation at $14,000 \mathrm{rpm}$ for 10 minutes and vacuum drying overnight at $50^{\circ} \mathrm{C}$. The $\mathrm{pH}$ of the nanoparticle solution was kept at 8.0.

\section{Characterization}

Ultraviolet-visible spectra for the deep red dispersions were measured using an ultraviolet 1650 PC-Shimadzu B spectrophotometer (Shimadzu, Osaka, Japan). Powder $\mathrm{X}$-ray diffraction experiments were carried out on a 6000 X-ray diffraction instrument (Shimadzu), and the patterns were recorded at a scan speed of $4^{\circ}$ per minute with $\mathrm{Cu} \mathrm{K} \alpha 1$ radiation $(\lambda=1.54060 \AA)$ operating at $40 \mathrm{kV}$ and $40 \mathrm{~mA}$. Morphological analysis of the samples was carried out using field emission scanning electron microscopy; the measurements were made on a JSM-7600F instrument (JEOL, Eching, München, Germany). Samples were dried and sputter-coated with gold film using an SCD005 sputter coater (Baltec, Canonsburg, PA, USA). The samples were then imaged under the field emission scanning electron microscope. Transmission electron microscopic observations were carried out using an H-7100 electron microscope (Hitachi, Tokyo, Japan), and the particle size distribution was obtained using UTHSCSA Image Tool version 3.0 software (The University of Texas Health Science Center, San Antonio, TX, USA). Molecular analysis of the samples was performed by Fourier transform infrared (FT-IR) spectroscopy using a series 100, 1650 spectrophotometer (Perkin-Elmer, Santa Clara, CA, USA), recorded over the range of $200-4,000 \mathrm{~cm}^{-1}$.

\section{Microorganisms}

Four species of bacteria, including two Gram-positive species, ie, a pathogenic strain of MRSA and B. subtilis, two Gram-negative species, ie, $S$. choleraesuis and
P. aeruginosa, and one yeast species (C. albicans) were obtained from the Microbial Culture Collection Unit, Institute of Bioscience, Universiti Putra Malaysia. Cultures were maintained on Luria-Bertani agar (Fluka, Buchs, Switzerland). Prior to incubation with the nanoparticles, the microorganisms were cultured overnight in $5 \mathrm{~mL}$ of Luria-Bertani broth (Fluka) in a Certomat BS-T incubation shaker (Sartorius Stedim Biotech, Aubagne, France) at $37^{\circ} \mathrm{C}$ and $150 \mathrm{rpm}$ until the microbial culture reached an $\mathrm{OD}_{600}$ of 1.0 , corresponding to $8 \times 10^{8}$ colony-forming units per $\mathrm{mL}$, as determined using an Ultrospec ultravioletvisible 3000 spectrophotometer (Amersham Pharmacia Biotech, Cambridge, UK). The antimicrobial activity of the chitosan-copper nanoparticles against the selected microorganisms was investigated by two methods, ie, qualitative evaluation using the zone of inhibition method, and quantitative evaluation.

\section{Antimicrobial susceptibility test}

The antibacterial properties of the as-synthesized chitosan-copper nanoparticles were evaluated by a qualitative method against the aforementioned microorganisms using the agar disk diffusion method as described previously. ${ }^{37}$ Gram-positive and Gram-negative bacteria were cultured on LB agar medium (Fluka) while yeast was cultured on potato dextrose agar (Becton Dickinson Difco, Franklin Lakes, NJ, USA). Briefly, $20 \mathrm{~mL}$ of liquid Mueller Hinton agar ( $\mathrm{pH} 7.3 \pm 0.2$ at $25^{\circ} \mathrm{C}$ ) was poured onto disposable sterilized Petri dishes and allowed to solidify. The surfaces of the solidified agar plates were allowed to dry in the incubator prior to streaking of microorganisms onto the surface of the agar plates. Next, $100 \mu \mathrm{L}$ of the microbial culture suspension in broth containing approximately $10^{6}$ colony-forming units per $\mathrm{mL}$ as measured spectrophotometrically was streaked over the dried surface of the agar plate and spread uniformly using a sterilized glass rod and allowed to dry before applying the loaded disks. The chitosan-copper nanoparticle compounds were suspended in sterilized distilled water, and blank sterilized Whatman No 1 filter paper disks were loaded with the suspension. The loaded disks were applied carefully to the surface of the seeded agar plates using sterile forceps. The experiment was carried out in triplicate and the diameters of the zones of inhibition were measured after 24 hours of incubation at $37^{\circ} \mathrm{C}$. Standard antimicrobial agents including nystatin (for yeast, $100 \mathrm{mg} / \mathrm{mL}$ ), ampicillin (for Gram-negative bacteria, $100 \mathrm{mg} / \mathrm{mL}$ ), and streptomycin (Gram-positive bacteria, $100 \mathrm{mg} / \mathrm{mL}$ ) were used as controls. 


\section{Effect of chitosan-copper nanoparticles on inhibition of microbial growth}

Growth studies with optical density (OD) measurements were used to evaluate the antimicrobial activity in a quantitative manner. Prior to incubation with the nanoparticles, the bacteria were cultured overnight in $5 \mathrm{~mL}$ of Luria-Bertani broth and the yeast was cultured in potato dextrose broth. The microbial culture suspension was adjusted to an $\mathrm{OD}_{600}$ of 1.0 as determined spectrophotometrically. The overnight cultures were diluted $10^{5}$ to approximately $10^{4}$ colony-forming units per $\mathrm{mL}$ using sterile broth for further investigation. The chitosan-copper nanoparticles were suspended in sterilized distilled water (Millipore) to give a final concentration of $2.5 \mathrm{mg}$ in each well, and the suspension was distributed uniformly on the surface of six-well sterile tissue culture plates (Nalge Nunc International, Roskilde, Denmark). To examine microbial growth and to determine growth behavior in the presence of the chitosan-copper nanoparticles, $100 \mu \mathrm{L}$ of the microbial culture suspensions were added to each well supplemented with the nanoparticle compounds. Cultures of nanoparticle-free medium under the same growth conditions were used as a control. To avoid potential optical interference caused by the light-scattering properties of the nanoparticles during determination of OD in the growing cultures, the same Luria-Bertani broth medium without microorganisms but containing the same concentration of nanoparticles cultured under the same conditions was used as the blank control. These plates, as well as the negative and the positive control plates, were incubated overnight in a Certomat SII incubation shaker at $37^{\circ} \mathrm{C}$ and in a humid atmosphere to minimize evaporation from each well. Following incubation of the test microorganisms with the nanoparticles overnight, the OD of the cultures in each well was determined spectrophotometrically. The corresponding number of colony-forming units was determined and the percentage inhibition was calculated as follows:

$$
\text { Inhibition rate }=1-\mathrm{OD}_{\text {sample }} / \mathrm{OD}_{\text {control }} \times 100
$$

The efficiency of the nanoparticles in inhibiting the growth of microorganisms was determined by differences in the equivalent number of colony-forming units before and after treatment as a percentage of microbes that were inhibited by the nanoparticles as calculated from the previous equation.

\section{Results and discussion}

As mentioned earlier, the effects of chitosan on the stability and antimicrobial properties of the synthesized chitosan-copper nanoparticles were evaluated. Prior to susceptibility testing, the synthesized nanoparticles were subjected to different methods of characterization to determine their purity. Samples containing various amounts of dispersant $(0.05,0.1,0.2$, or $0.5 \mathrm{wt} \%)$ differed with regard to the color of the nanoparticles obtained, ie, from light brown to deep red. This may be indicative of particle stability, as evidenced by the characterization methods. Nevertheless, the samples containing various chitosan concentrations did not display any significant difference in color throughout the different stages of the reaction. The green coloration of the chitosan-copper complex, ${ }^{23}$ obtained by addition of sodium hydroxide, did not differ over the $0.05-0.5 \mathrm{wt} \%$ range. A different pattern was observed for particle sizes and antimicrobial properties, with slight variation in susceptibility of the $0.2 \mathrm{wt} \%$ and $0.5 \mathrm{wt} \%$ nanoparticles.

The surfaces of chitosan-copper nanoparticles are covered by fragments of chitosan which protect against aggregation and oxidation. ${ }^{38}$ The nuclei of the individual nanocrystals are attracted to each other by weak van der Waals forces, and the stabilizer provides insulation between the particles by overcoming these forces, a phenomenon seen with both polymers and surfactants. ${ }^{38,39}$ Interestingly, this influence was noticed in almost all aspects of our research, including in the antimicrobial susceptibility test. For instance, the surface plasmon resonance peaks of the red samples were obtained immediately after synthesis. These samples showed a band at $582 \mathrm{~nm}$, as shown in Figure 1. The peaks seen are features of chitosan-copper nanoparticles, which are known to show absorbance in the range of $500-600 \mathrm{~nm} \cdot .^{40}$ As observed, the

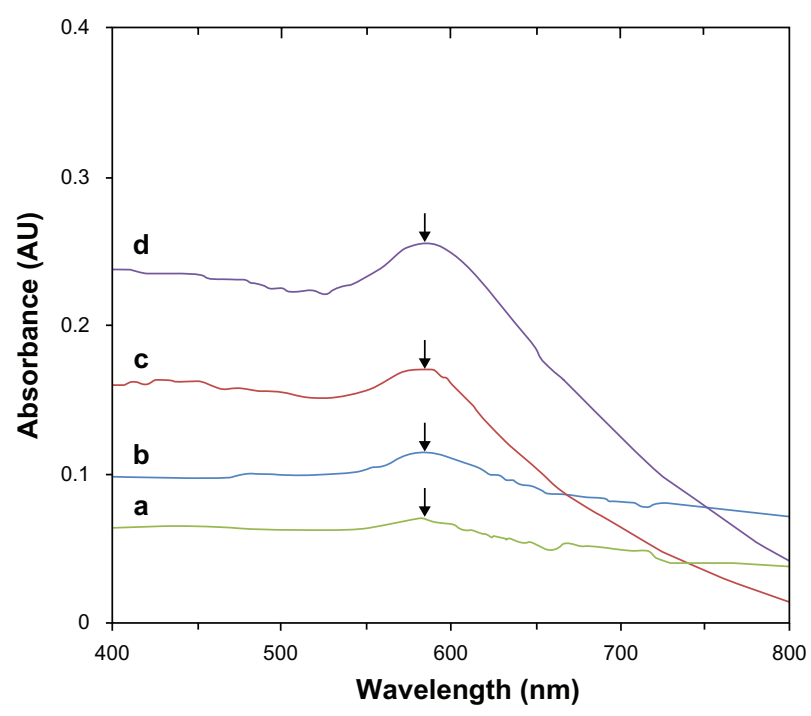

Figure I Ultraviolet-visible spectra of chitosan-copper nanoparticles at different concentrations of chitosan $(0.05,0.1,0.2$, and 0.5 wt $\%$ [a-d], respectively). 
relative absorbance of the samples increased simultaneously with the increase in concentration of chitosan from $0.05 \mathrm{wt} \%$ to $0.5 \mathrm{wt} \%$. This suggests that the size of the chitosan-copper nanoparticles decreased with increasing stabilizer concentrations, with $0.5 \mathrm{wt} \%$ having the smallest size. The higher peak absorbance for the $0.5 \mathrm{wt} \%$ concentration indicate greater dispersion of the particles..$^{23}$ Further, the stability of the particles increased from low to high concentration. The low surface plasmon resonance peak intensity noted in the $0.05 \mathrm{wt} \%$ sample indicates low defragmentation of molecules, which in turn resulted in less capping of the nanoparticles. The result obtained is consistent with that for the $\mathrm{X}$-ray diffraction patterns for the nanoparticles, as shown in Figure 2. The peaks at $36.79^{\circ}, 43.49^{\circ}, 50.65^{\circ}$, and $74.15^{\circ}$ of the spectrum correspond to the (111), (111), (200), and (220) respectively, which represent crystal face-centered cubic (fcc) of copper (Cu X-ray diffraction reference number 01-089-2838) ${ }^{39}$ The peak at 19.76 is due to the presence of chitosan in the nanoparticles. As observed in spectra $(\mathrm{a}-\mathrm{d})$ in Figure 2, the peak intensity reduced with decreasing chitosan concentration. This indicates an interaction between the nanoparticles and the stabilizing medium. No other $\mathrm{CuO}$ or $\mathrm{Cu}_{2} \mathrm{O}$ impurity peaks were observed in any of the spectra, suggesting that the synthesized particles were of high purity. An estimate of nanoparticle size was done using
Sherrer's equation (equation 2) and found to be in the range of $10-15 \mathrm{~nm}$. It is known that copper nanoparticles rapidly oxidize on exposure to the atmosphere, which can result in particle aggregation ${ }^{23}$ and could affect the antimicrobial properties of chitosan-copper nanoparticles. Interestingly, nanoparticles stabilized by chitosan in this study showed antimicrobial activity according to the polymer content of the particles, which confirms the stabilizing effect of the chitosan used. The Sherrer's formula is presented in equation 2 :

$$
\mathrm{d}=\frac{\mathrm{K} \lambda}{\beta \cos \theta}
$$

where $\mathrm{d}$ is the mean nanoparticle diameter, $\lambda$ is $1.5418 \AA$ (wavelength of the radiation source), $\mathrm{K}$ is the Scherer's constant or shape factor with a value of $0.9, \theta$ is the Bragg angle, and $\beta_{1 / 2}$ is the width of peak at half height. ${ }^{23}$

An additional confirmatory test was performed by studying the molecular interaction between the chitosan medium and the synthesized nanoparticles using FT-IR. Figure 3a-e indicates the spectra of pure chitosan and the chitosan-mediated copper nanoparticles at different concentrations $(0.05-0.5 \mathrm{wt} \%)$. The FT-IR spectra for chitosan (a) shows vibration bands at $3,358 \mathrm{~cm}^{-1}$, which may be due to overlapping of $\mathrm{O}-\mathrm{H}$ and amine $\mathrm{N}-\mathrm{H}$ stretching bands; the

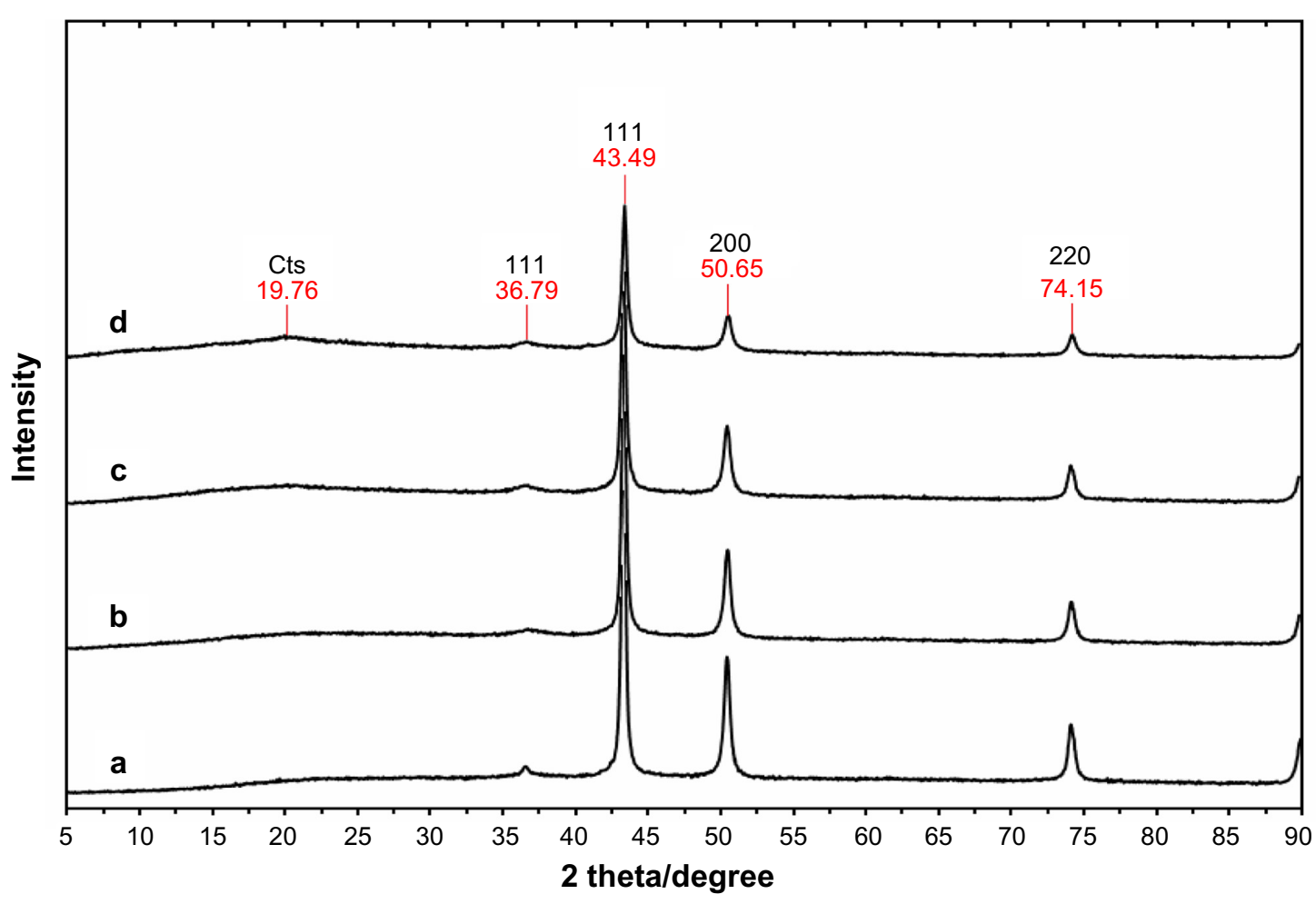

Figure 2 X-ray diffraction patterns of chitosan-copper nanoparticles at different concentrations of chitosan $(0.05,0.1,0.2$, and 0.5 wt $\%$ [a-d], respectively). 


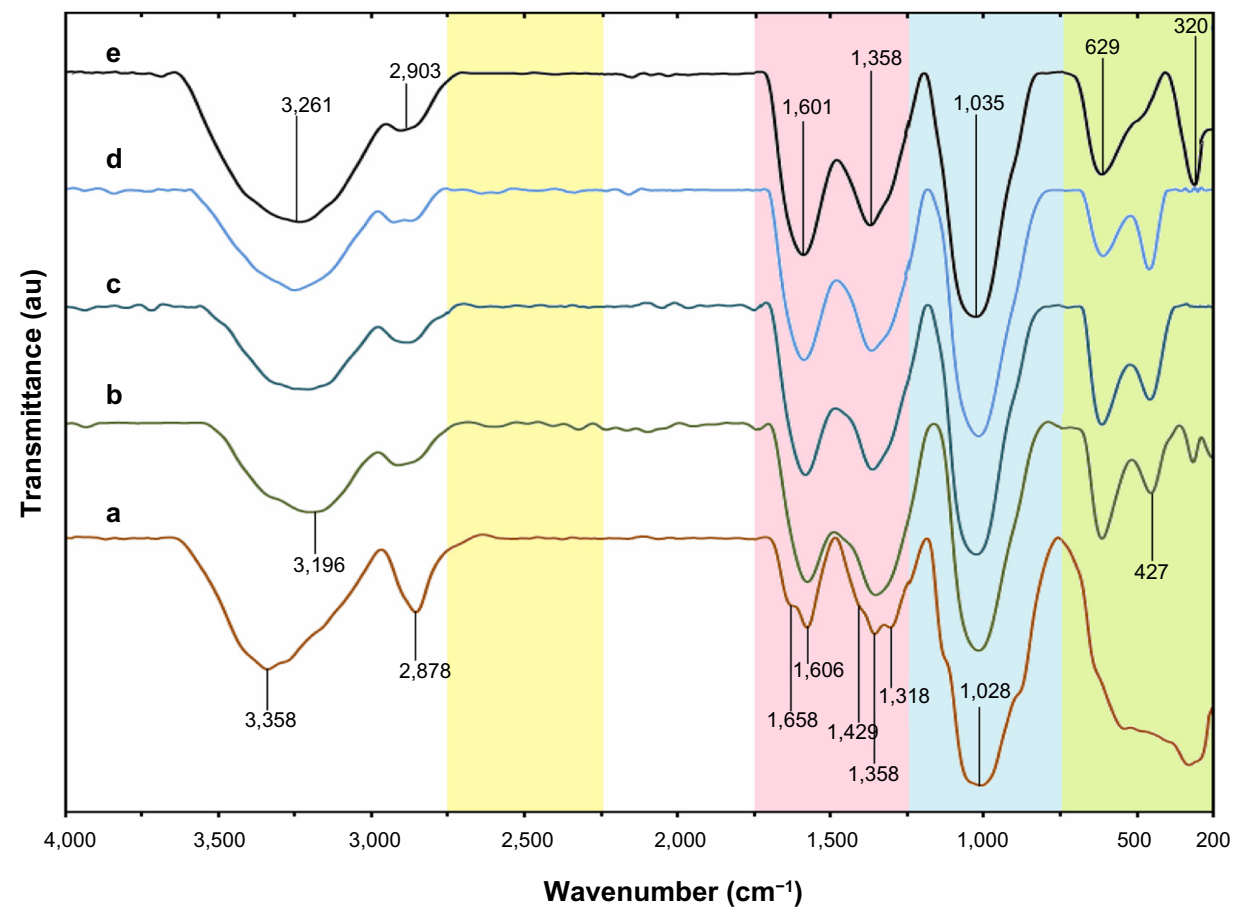

Figure 3 Fourier transform infrared spectra of chitosan (a) and chitosan-copper nanoparticles $(0.05,0.1,0.2$, and 0.5 wt\% [b-e], respectively).

peak at 2,878 $\mathrm{cm}^{-1}$ indicates aliphatic $\mathrm{C}-\mathrm{H}$ stretching; 1,658 and $1,606 \mathrm{~cm}^{-1}$ indicates $\mathrm{N}-\mathrm{H}$ bending; $1,429,1,358$, and $1,318 \mathrm{~cm}^{-1}$ indicates $\mathrm{C}-\mathrm{H}$ bending; and $1,028 \mathrm{~cm}^{-1}$ indicates $\mathrm{C}-\mathrm{O}$ stretching..$^{41}$ For spectra b-e in Figure 3, blue shifts and decreased intensity of peaks were noticed, eg, a blue shift of the $\mathrm{N}-\mathrm{H}$ bending peak at $1,606 \mathrm{~cm}^{-1}$ to $1,601 \mathrm{~cm}^{-1}$; however, the second $1,658 \mathrm{~cm}^{-1}$ peak was missing in all the spectra. This confirms capping of the copper nanoparticle surface by the $\mathrm{N}-\mathrm{H}$ groups of the polymer. Similarly, peaks at 1,429 to $1,318 \mathrm{~cm}^{-1}$ were not observed. In addition, new moderate intensity peaks representing copper nanoparticles were evident at $629 \mathrm{~cm}^{-1}$ for all the samples, and at $427 \mathrm{~cm}^{-1}$ for the $0.05 \%, 0.1 \%$, and $0.2 \%$ concentrations (b-d) and at $320 \mathrm{~cm}^{-1}$ for the $0.05 \%$ and $0.5 \mathrm{wt} \%$ concentrations (b and e). The two vibration bands could also indicate an interaction between the copper nanoparticles and the chitosan medium.

A micrograph of the chitosan stabilized copper nanoparticles (Figure 4) indicates the size of the nanoparticles at different concentrations. The size of the nanoparticles appeared to decrease with increasing concentration of the dispersant. This is consistent with the results obtained from other characterizations conducted on the nanoparticles. The $0.05 \mathrm{wt} \%$ chitosan-copper nanoparticles were found to be larger, ranging from $50 \mathrm{~nm}$ to $300 \mathrm{~nm}$ with a standard deviation of $186.24 \pm 69.21 \mathrm{~nm}$ (Figure 4A). Subsequent nanoparticles with a concentration of $0.1 \mathrm{wt} \%$ were observed to be slightly smaller, with a size range of $50-270 \mathrm{~nm}$. In the same manner, chitosan-copper nanoparticles with a concentration of $0.2 \mathrm{wt} \%$ had a size range of 5-50 nm and a standard deviation of $18.29 \pm 7.75 \mathrm{~nm}$. The chitosancopper nanoparticles with $0.5 \mathrm{wt} \%$ chitosan appeared to have the lowest particle size, being as low as $2 \mathrm{~nm}$ with a standard deviation of $9.61 \pm 11.90 \mathrm{~nm}$. The low standard deviation seen for the $0.2 \mathrm{wt} \%$ chitosan is due to the higher nanoparticle size distribution in the sample, as observed in the histogram (Figure 4). This emphasizes the relevance of chitosan in controlling the size of the nanoparticles, as described in our previous research. ${ }^{23}$ In addition, it is noteworthy that the morphology observed in the transmission electron micrographs for $0.1 \mathrm{wt} \%$ chitosan was rod-shaped or cylindrical, which was not observed in the field emission scanning electron micrographs of the same sample. The number of nanoparticles counted for transmission electron microscopy was 250 per hour of stirring time.

The images obtained for the various concentrations of the stabilized crystals indicate that the copper nanoparticles were embedded within the matrix of the polymer. Typical micrographs for the nanoparticles are seen in Figure 5, showing particles of different diameters that are polydispersed. The particles were observed to be polydispersed and distributed. Although the copper nanoparticles appear to be predominantly spherical in shape, the particle size and distribution patterns vary according to the chitosan concentrations $(0.05-0.5 \mathrm{wt} \%[\mathrm{~A}-\mathrm{D}])$. Figure $5 \mathrm{~A}$ and $\mathrm{B}$ 

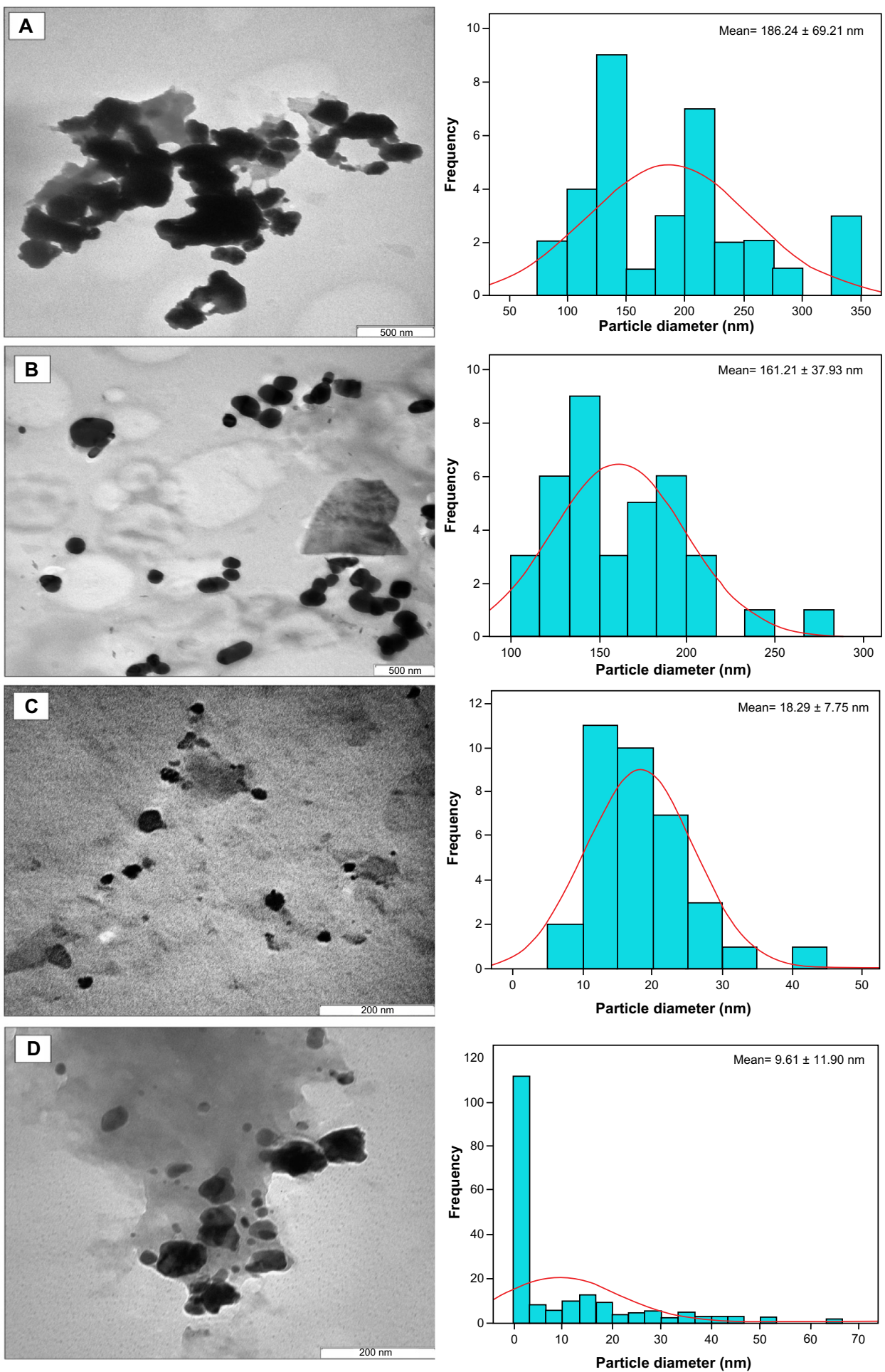

Figure 4 Transmission electron micrographs of chitosan-copper nanoparticles at different concentrations of chitosan medium, $0.05,0.1,0.2$ and 0.5 wt\%, (A-D) respectively. Notes: Attached to each micrograph, is the size bar chart fitted with Gaussian curve which demonstrates the size distribution pattern of the nanoparticles in the micrographs. The standard mean sizes of the nanoparticles were also determined through Gaussian curve.

showing the $0.05 \%$ and $0.1 \mathrm{wt} \%$ concentrations indicate larger-sized nanoparticles and higher agglomeration, with the nanoparticles forming large flocks of aggregated particles. However, the nanoparticles synthesized at higher chitosan concentrations $(0.2 \%$ and $0.5 \mathrm{wt} \%)$ had relative smaller sizes and a more uniform distribution (Figure 5C and D).

These findings accentuate the important role of the polymer as a stabilizer. It is known that copper nanoparticles tend to agglomerate on synthesis due to the high tendency of 

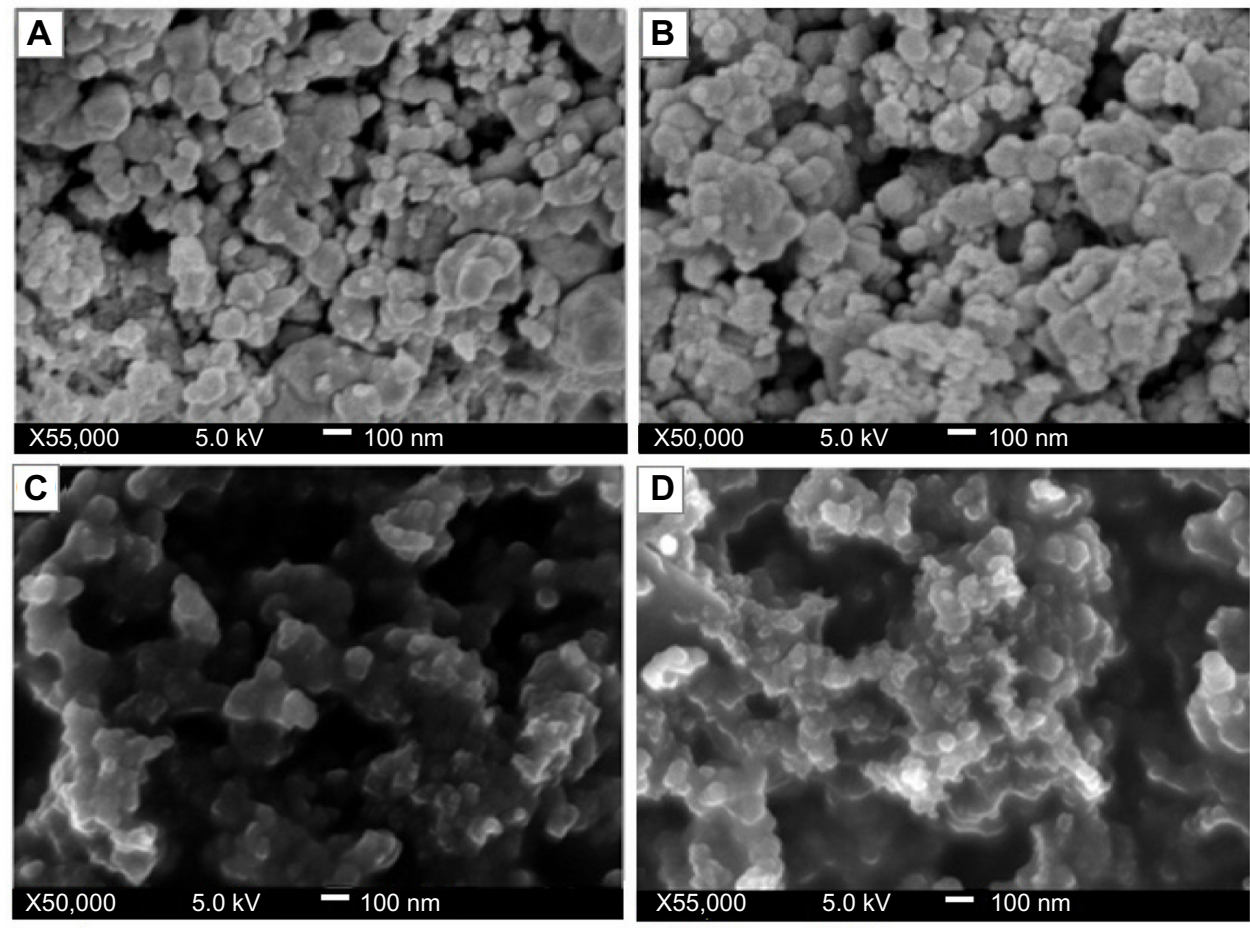

Figure 5 Field emission scanning electron micrographs of chitosan-copper nanoparticles at different concentration of chitosan medium, $0.05,0.1,0.2$ and 0.5 wt\% (A-D), respectively.

copper nuclei to bond. The aggregation may also be due to the high surface area of the copper nanoparticles. ${ }^{23}$

The chitosan stabilized copper nanoparticles exhibited both antibacterial and antifungal activity against Gram-positive bacteria, Gram-negative bacteria, and yeast. The chitosan medium supported the efficiency of the copper nanoparticles. As shown in Figure 6A-E, the antimicrobial activity (indicated by the zones of inhibition) of chitosan-copper nanoparticle compounds 1 (0.05 wt\%), 2 (0.1 wt\%), $3(0.2 \mathrm{wt} \%)$, and 4 $(0.5 \mathrm{wt} \%)$, respectively, was clearly observed in all the samples.

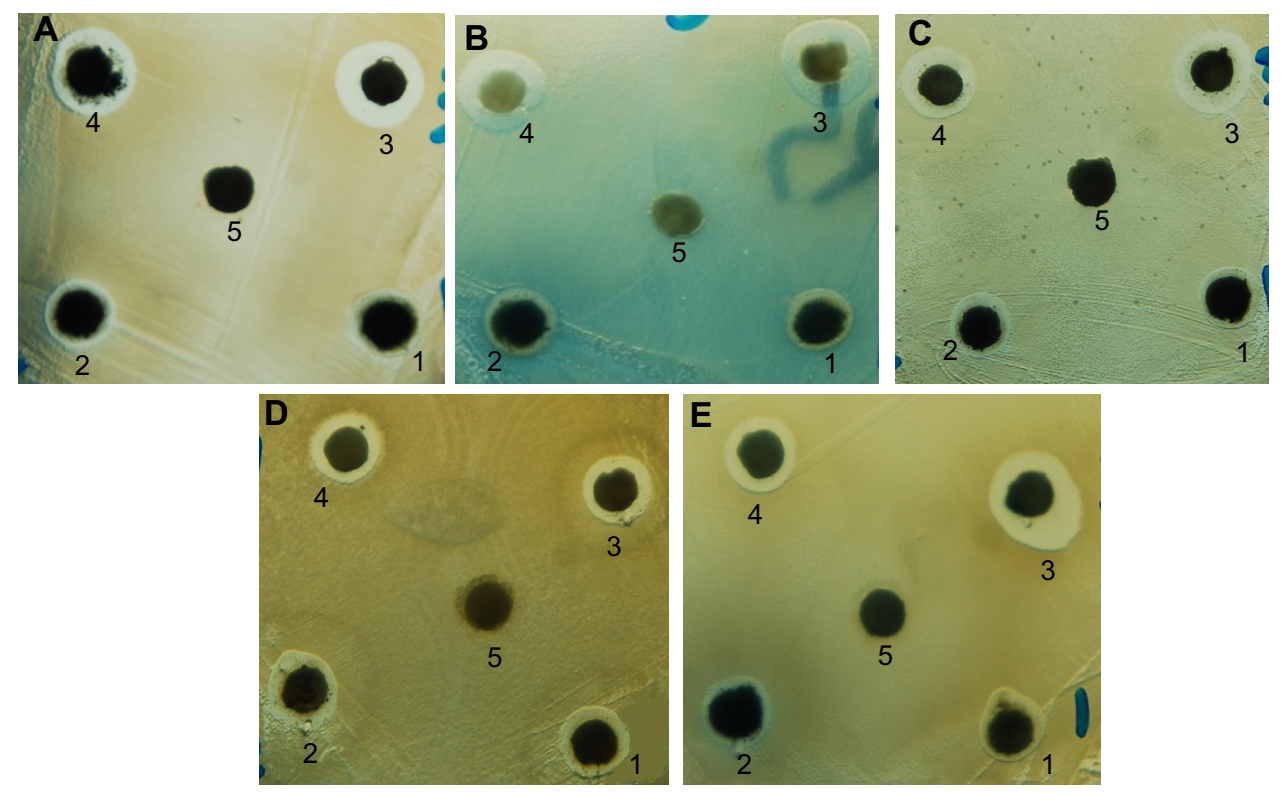

Figure 6 Antimicrobial activity of chitosan-copper nanoparticle compounds I (0.05 wt\%), 2 (0.I wt\%), 3 (0.2 wt\%), and 4 (0.5 wt\%), and 5 (chitosan) against bacteria and yeast using the disk agar diffusion method. Photographs of chitosan-copper nanoparticles and (A) methicillin-resistant Staphylococcus aureus, (B) Pseudomonas aeruginosa, (C) Salmonella choleraesuis, (D) Bacillus subtilis, and (E) Candida albicans. 
Table I Antimicrobial activity of copper-chitosan nanoparticles against different microorganisms as determined by the disk agar diffusion method*

\begin{tabular}{llllll}
\hline NPs/MO & BS & MRSA & PA & SC & CA \\
\hline Compound I & 9 & 9 & 8 & 9 & 9 \\
Compound 2 & 10 & 9 & 11 & 9 & 9 \\
Compound 3 & 9 & 11 & 12 & 11 & 11 \\
Compound 4 & 9 & 11 & 10 & 10 & 9 \\
Control** & 23 & 23 & 22 & 25 & 22
\end{tabular}

Notes: *Diameters of zones of inhibition were measured to nearest $\mathrm{mm}$; **control (ampicillin [Gram-negative], streptomycin [Gram-positive], and nystatin [yeast]). Abbreviations: NPs, nanoparticles (I [0.05 wt\%], 2 [0.I wt\%], 3 [0.2 wt\%], and 4 [0.5 wt\%]); MO, microorganism; BS, Bacillus subtilis; MRSA, methicillin-resistant Staphylococcus aureus; PA, Pseudomonas aeruginosa; SC, Salmonella choleraesuis; CA, Candida albicans.

Further, chitosan did not demonstrate any zone of inhibition, suggesting a lack of antimicrobial activity of chitosan at $\mathrm{pH}$ 7.4 which is corresponding to the $\mathrm{pH}$ of the Mueller-Hinton agar medium used in the experiment. This is in accordance with the fact that chitosan has antimicrobial activity only in an acidic medium because of its poor solubility above $\mathrm{pH} 6.5 .{ }^{42,43}$ The antimicrobial activity of chitosan depends on several factors, including its degree of polymerization, molecular weight, nutrient composition, host, natural nutrient constituency, solvent, target microorganism, and physicochemical properties, and is inversely affected by $\mathrm{pH} .{ }^{43}$ However, it is noteworthy that the zone of inhibition of the particles is generally increased when the chitosan medium is increased.

Numbers shown in Table 1 represent the diameter $(\mathrm{mm})$ of the zones of inhibition measured to the nearest mm using a caliper. The zone of inhibition diameter was highest for compound 3 , which is the $0.2 \mathrm{wt} \%$ concentration (Figure 7). The results for microbial growth inhibition according to OD measurements (Figure 8A-C) support the results obtained by the agar diffusion method for the nanoparticles tested. All the chitosan-copper nanoparticle compounds showed high inhibition rates against the tested microorganisms (see Table 2) suggesting that these nanoparticles are effective antibacterial and antifungal agents.

This indicates slower diffusion of the nanoparticles released from the chitosan medium in the sample, which is responsible for the antibacterial activity. In addition, the nanoparticles in the $0.2 \mathrm{wt} \%$ concentration, although larger than the nanoparticles in the $0.5 \mathrm{wt} \%$ concentration, have a better smaller-sized nanoparticle distribution, as observed in the transmission electron microscopic analysis, which contributed to their higher antimicrobial activity. This observation also indicates the optimum concentration of stabilizer for the antimicrobial analysis. It is generally assumed that nanoparticles stabilized by biopolymers have the advantage of a prolonged release time, which improves their antimicrobial properties. ${ }^{44}$ The controlled-release profile of the nanoparticles in antimicrobial susceptibility tests is yet to be determined. The results of the present study are in agreement with other reports that indicate greater activity of copper nanoparticles against Gram-negative microorganisms. ${ }^{44}$ Our efficacy results indicate that the chitosan-stabilized nanocrystals are more active against $P$. aeruginosa, a Gramnegative microorganism. This can partially be explained by the facilitated influx of smaller-sized nanoparticles into the cell wall of Gram-negative bacteria which consists of

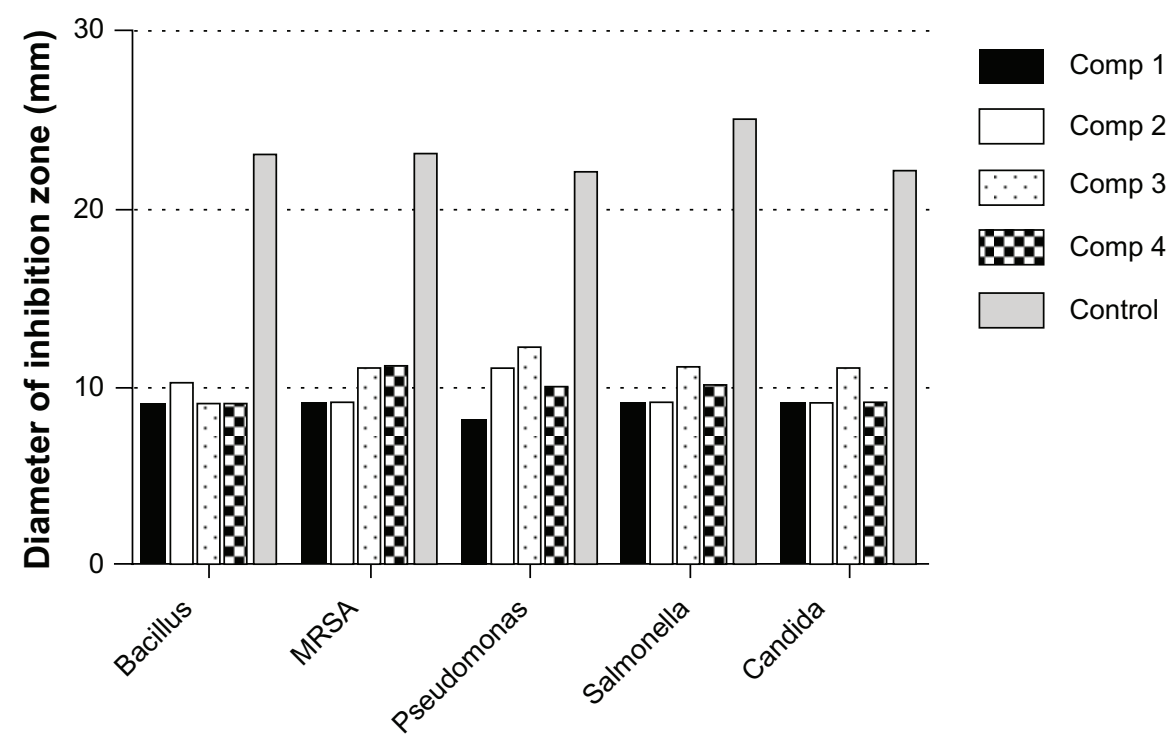

Figure 7 Diameter of inhibitory zones for copper-chitosan nanoparticle compounds I ( 0.05 wt\%), 2 ( 0.1 wt $\%)$, 3 ( 0.2 wt $\%)$, and 4 ( 0.5 wt $\%)$ against bacteria and yeast, along with the control antimicrobial agents (ampicillin for Gram-negative organisms, streptomycin for Gram-positive organisms, and nystatin for yeast).

Abbreviations: MRSA, methicillin-resistant Staphylococcus aureus; Comp, compound. 

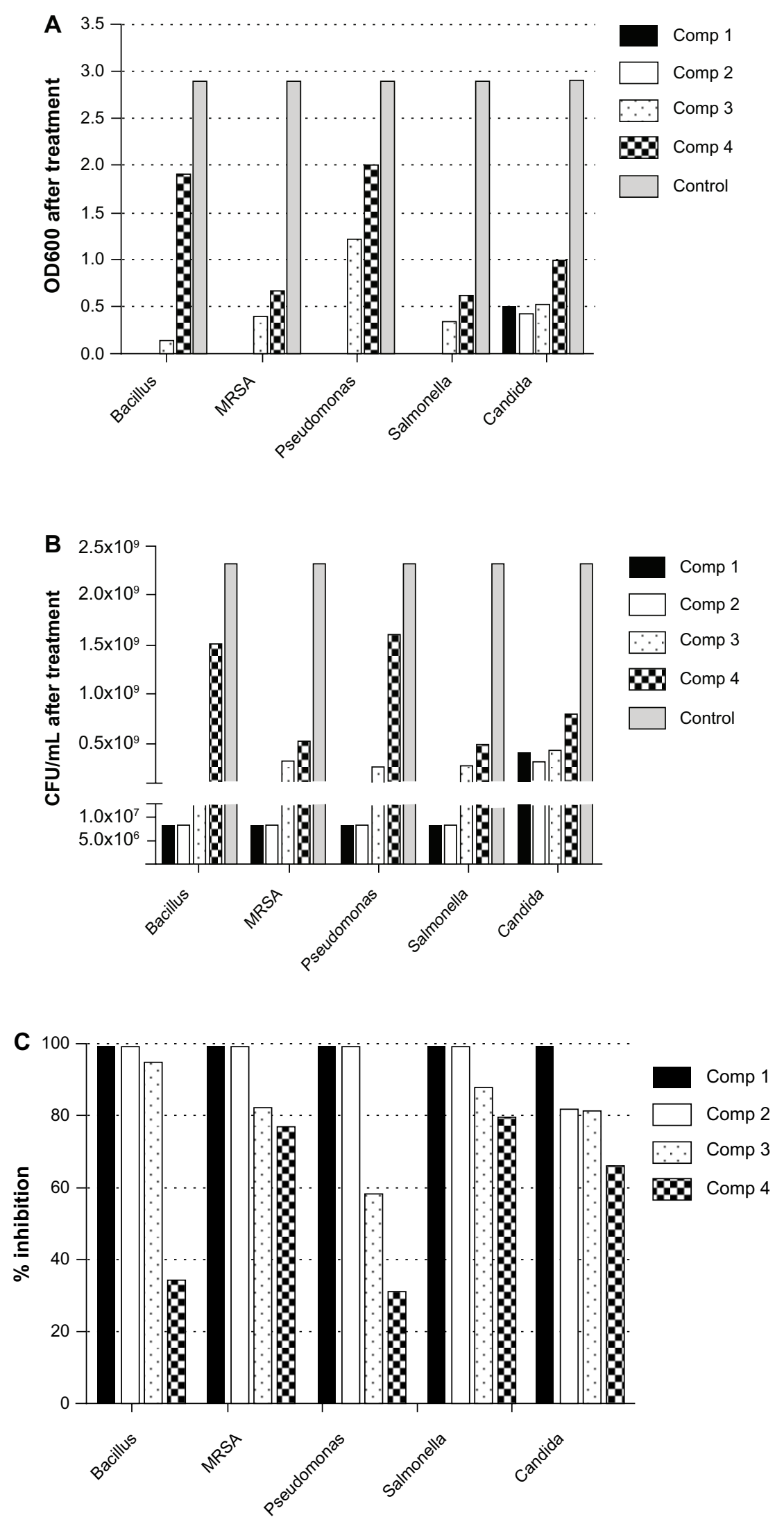

Figure 8 Effect of chitosan-copper nanoparticles on inhibition of growth of microorganisms using $\mathrm{OD}_{600}$ measurements. $(\mathbf{A})$ and $(\mathbf{B}) \mathrm{OD}_{600}$ and corresponding calculated numbers of CFU per $\mathrm{mL}$ of each microorganism following treatment with nanoparticle compounds I (0.05 wt \%), $2(0.1$ wt \%), 3 (0.2 wt\%), and 4 (0.5 wt\%). (C) Inhibition rate (\%) calculated from equation (I) of each nanoparticle compound against bacteria and yeast.

Abbreviations: CFU, colony-forming units; OD, optical density; MRSA, methicillin-resistant Staphylococcus aureus; Comp, compound. 
Table 2 Inhibition rate (\%) of chitosan-copper nanoparticles against different microorganisms

\begin{tabular}{llllll}
\hline NPs/MO & BS & MRSA & PA & SC & CA \\
\hline Compound I & 99.65 & 99.65 & 99.65 & 99.65 & 82.75 \\
Compound 2 & 99.65 & 99.65 & 99.65 & 99.65 & 82.2 \\
Compound 3 & 95.17 & 82.2 & 58.62 & 88.27 & 81.37 \\
Compound 4 & 34.48 & 77.58 & 31.03 & 79.31 & 65.86 \\
\hline
\end{tabular}

Abbreviations: NPs, nanoparticles (I [0.05 wt\%], 2 [0.1 wt\%], 3 [0.2 wt\%], and 4 [0.5 wt\%]); MO, microorganism; BS, Bacillus subtilis; MRSA, methicillin-resistant Staphylococcus aureus; PA, Pseudomonas aeruginosa; SC, Salmonella choleraesuis; CA, Candida albicans.

a unique outer membrane layer and a single peptidoglycan layer as compared to the cell wall of Gram-positive bacteria with several peptidoglycan layers..$^{45,46}$ Thus, the cell wall is more exposed to nanoparticles through the outer bacterial membrane. The unique high surface to volume ratio of chitosan-copper nanoparticles enables them to interact with the bacterial cell membrane through its surface, ${ }^{10}$ which leads to the death of the bacterium. ${ }^{47}$ Therefore, the size of the nanoparticles is important for antimicobial activity.

\section{Conclusion}

We synthesized high-purity metallic chitosan-copper nanoparticles via a chemical method. The antimicrobial activity of the nanoparticles was determined according to the chitosan concentration using a variety of bacterial species and a fungal species. The $0.2 \mathrm{wt} \%$ concentration was determined to be optimal, due to its higher activity against the microbial species tested. Transmission electron micrographs for the $0.5 \mathrm{wt} \%$ concentration indicate the size of the nanoparticles to be $2 \mathrm{~nm}$. Our results indicate the future potential of these chitosan-copper nanoparticles for combating pathogenic microorganisms. Further in vivo studies to determine the toxicity of these nanomaterials will allow for the application and use of these nanoparticles, which can be prepared in a simple and cost-effective manner and may be suitable for formulation of new types of antimicrobial materials for pharmaceutical and biomedical applications, such as antimicrobial next-to-skin fabrics.

\section{Acknowledgments}

The authors would like to thank Rafiuz Zaman Haroun from the microscopy unit of the Institute of Bioscience, Universiti Putra Malaysia, for technical assistance with the microscopic characterization aspects of this research. The authors would like to thank Universiti Putra Malaysia for the support of Dr Kamyar Shameli under postdoctoral program and Mr Muhammad Sani Usman under a doctoral graduate scholarship program. The authors are also grateful to the Malaysian International Scholarship, Ministry of Higher
Education, Malaysia, for sponsorship of Dr Mohamed Ezzat El Zowalaty, under a postdoctoral scholarship award.

\section{Disclosure}

The authors report no conflicts of interest in this work.

\section{References}

1. Raffi M, Mehrwan S, Bhatti TM, et al. Investigations into the antibacterial behavior of copper nanoparticles against Escherichia coli. Ann Microbiol. 2010;60:75-80.

2. Lee C-R, Cho IH, Jeong BC, Lee SH. Strategies to Minimize Antibiotic Resistance. Int J Environ Res Public Health. 2013;10(9):4274-4305.

3. El Zowalaty ME. Alarming trend of antibiotic resistance in Pseudomonas aeruginosa isolates. Journal of Pure and Applied Microbiology. 2012;6(1):175-183.

4. Dîaz-Visurraga J, Daza C, Pozo C, et al. Study on antibacterial alginate-stabilized copper nanoparticles by FTIR and 2D-IR correlation spectroscopy. Int J Nanomedicine. 2012;7:3597-3612.

5. Janardhanan R, Karuppaiah M, Hebalkar N, Rao TN. Synthesis and surface chemistry of nano silver particles. Polyhedron. 2009;28: 2522-2530.

6. Bhattacharyya S, Kudgus RA, Bhattacharya R, Mukherjee P. Inorganic nanoparticles in cancer therapy. Pharm Res. 2011;28:237-259.

7. Lim ZZ, Li JE, Ng CT, Yung LY, Bay BH. Gold nanoparticles in cancer therapy. Acta Pharmacol Sin. 2011;32:983-990.

8. Hirsch LR, Stafford R, Bankson J, et al. Nanoshell-mediated nearinfrared thermal therapy of tumors under magnetic resonance guidance. Proc Natl Acad Sci U S A. 2003;100:13549-13554.

9. Gyawali R, Ibrahim SA, Abu Hasfa SH, Smqadri SQ, Haik Y. Antimicrobial activity of copper alone and in combination with lactic acid against Escherichia coli O157:H7 in laboratory medium and on the surface of lettuce and tomatoes. J Pathog. 2011;2011: 650968 .

10. Chatterjee AK, Sarkar RK, Chattopadhyay AP, Aich P, Chakraborty R, Basu T. A simple robust method for synthesis of metallic copper nanoparticles of high antibacterial potency against E. coli. Nanotechnology. 2012;23:1-11.

11. Cho KH, Park JE, Osaka T, Park SG. The study of antimicrobial activity and preservative effects of nanosilver ingredient. Electrochim Acta. 2005;51:956-960.

12. Jung WK, Koo HC, Kim KW, Shin S, Kim SH, Park YH. Antibacterial activity and mechanism of action of the silver ion in Staphylococcus aureus and Escherichia coli. Appl Environ Microbiol. 2008;74: 2171-2178.

13. Durán N, Marcato PD, De Souza GI, Alves OL, Esposito E. Antibacterial effect of silver nanoparticles produced by fungal process on textile fabrics and their effluent treatment. J Biomed Nanotechnol. 2007;3:203-208

14. Gaggelli E, Kozlowski H, Valensin D, Valensin G. Copper homeostasis and neurodegenerative disorders (Alzheimer's, prion, and Parkinson's diseases and amyotrophic lateral sclerosis). Chem Rev. 1995;106: 1995-2044.

15. Lutsenko S, Barnes NL, Bartee MY, Dmitriev OY. Function and regulation of human copper-transporting ATPases. Physiol Rev. 2007;87: 1011-1046.

16. Ren G, Hu D, Cheng EW, Vargas-Reus MA, Reip P, Allaker RP. Characterisation of copper oxide nanoparticles for antimicrobial applications. Int J Antimicrob Agents. 2009;33:587-590.

17. Ruparelia JP, Chatterjee AK, Duttagupta SP, Mukherji S. Strain specificity in antimicrobial activity of silver and copper nanoparticles. Acta Biomater. 2008;4:707-716.

18. Wei Y, Chen S, Kowalczyk B, Huda S, Gray TP, Grzybowski BA. Synthesis of stable, low-dispersity copper nanoparticles and nanorods and their antifungal and catalytic properties. J Phys Chem C. 2010;114:15612-15616. 
19. Shameli K, Ahmad BM, Jaffar Al-Mulla EA, et al. Green biosynthesis of silver nanoparticles using Callicarpa maingayi stem bark extraction. Molecules. 2012;17:8506-8517.

20. Balazs AC, Emrick T, Russell TP. Nanoparticle polymer composites: where two small worlds meet. Science. 2006;314:1107-1110.

21. Shenhar R, Norsten TB, Rotello VM. Polymer-mediated nanoparticle assembly: structural control and applications. Adv Mater. 2005;17: 657-669.

22. Cho MS, Park SY, Hwang JY, Choi HJ. Synthesis and electrical properties of polymer composites with polyaniline nanoparticles. Mater Sci Eng C. 2004;24:15-18.

23. Usman MS, Ibrahim NA, Shameli K, Zainuddin N, Yunus WM. Copper nanoparticles mediated by chitosan: synthesis and characterization via chemical methods. Molecules. 2012;17:14928-14936.

24. Salavati-Niasari M, Davar F. Synthesis of copper and copper (I) oxide nanoparticles by thermal decomposition of a new precursor. Mater Lett. 2009;63:441-443.

25. Raveendran P, Fu J, Wallen SL. Completely "green" synthesis and stabilization of metal nanoparticles. J Am Chem Soc. 2003;125: 13940-13941.

26. Nemamcha A, Rehspringer JL, Khatmi D. Synthesis of palladium nanoparticles by sonochemical reduction of palladium (II) nitrate in aqueous solution. J Phys Chem B. 2006;110:383-387.

27. Ponce AA, Klabunde KJ. Chemical and catalytic activity of copper nanoparticles prepared via metal vapor synthesis. J Mol Catal A Chem. 2005;225:1-6.

28. Ohde H, Hunt F, Wai CM. Synthesis of silver and copper nanoparticles in a water-in-supercritical-carbon dioxide microemulsion. Chem Mater. 2001;13:4130-4135.

29. Giusti A, Giorgetti E, Laza S, Marsili P, Giammanco F. Multiphoton fragmentation of PAMAM G5-capped gold nanoparticles induced by picosecond laser irradiation at $532 \mathrm{~nm}$. J Phys Chem C. 2007;111: 14984-14991.

30. Joshi S, Patil S, Iyer V, Mahumuni S. Radiation induced synthesis and characterization of copper nanoparticles. Nanostruct Mater. 1998;10: 1135-1144

31. Dash M, Chiellini F, Ottenbrite R, Chiellini E. Chitosan A versatile semi-synthetic polymer in biomedical applications. Prog Polym Sci. 2011;36: 981-1014.

32. Esteban-Cubillo A, Pecharromán C, Aguilar E, et al. Antibacterial activity of copper monodispersed nanoparticles into sepiolite. J Mater Sci. 2006;41:5208-5212.

33. Qi L, Xu Z, Jiang X, Hu C, Zou X. Preparation and antibacterial activity of chitosan nanoparticles. Carbohydr Res. 2004;339:2693-2700.
34. Yoon KY, Hoon Byeon J, Park JH, Hwang J. Susceptibility constants of Escherichia coli and Bacillus subtilis to silver and copper nanoparticles. Sci Total Environ. 2007;373:572-575.

35. Du WL, Niu SS, Xu YL, et al. Antibacterial activity of chitosan tripolyphosphate nanoparticles loaded with various metal ions. Carbohydr Polym. 2009;75:385-389.

36. Trapalis C, Kokkoris M, Perdikakis G, et al. Study of antibacterial composite $\mathrm{Cu} / \mathrm{SiO} 2$ thin coatings. J Sol-Gel Sci Techn. 2003;26: 1213-1218.

37. Andrews JM; BSAC Working Party On Susceptibility Testing. BSAC standardized disc susceptibility testing method. J Antimicrob Chemother. 2001;48 Suppl 1:43-57.

38. Huang NM, Radiman S, Lim HN, Khiew PS, Chiu WS, Lee KH, Syahida A, Hashim R, Chia CH. $\gamma$-Ray assisted synthesis of silver nanoparticles in chitosan solution and the antibacterial properties. Chem Eng J. 2009;155:499-507.

39. Wu SH, Chen DH. Synthesis of high-concentration $\mathrm{Cu}$ nanoparticles in aqueous CTAB solutions. J Colloid Interface Sci. 2004;273: 165-169.

40. Mallick K, Witcomb MJ, Scurrell MS. In situ synthesis of copper nanoparticles and poly (o-toluidine): a metal-polymer composite material. Eur Polym J. 2006;42:670-675.

41. Shameli K, Ahmad MB, Yunus WM, et al. Green synthesis of silver/ montmorillonite/chitosan bionanocomposites using the UV irradiation method and evaluation of antibacterial activity. Int J Nanomedicine. 2010;5:875-887.

42. Goy RC, de Britto DD, Assis OB. A review of the antimicrobial activity of chitosan. Polímeros: Ciência e Tecnologia. 2009;19:241-247.

43. Rabea EI, Badawy ME, Stevens CV, Smagghe G, Steurbaut W. Chitosan as antimicrobial agent: applications and mode of action. Biomacromolecules. 2003;4:1457-1465.

44. Longano D, Ditaranto N, Sabbatini L, Torsi L, Cioffi N. Synthesis and antimicrobial activity of copper nanomaterials. Nano-Antimicrobials: Progress and Prospects. 2012;3:85-117.

45. Hajipour MJ, Fromm KM, Ashkarran AA, Jimenez De Aberasturi D, de Larramendi IR, Rojo T, Serpooshan V, Parak WJ, Mahmoudi M. Antibacterial properties of nanoparticles. Trends Biotechnol. 2012;30(10):497-554.

46. Silhavy TJ, Kahne D, Walker S. The bacterial cell envelope. Cold Spring Harb Perspect Biol. 2010;2(5):a000414.

47. Theivasanthi T, Alagar M. Studies of copper nanoparticles effects on microorganisms. Ann Bio Res. 2011;2:368-373.
Video abstract

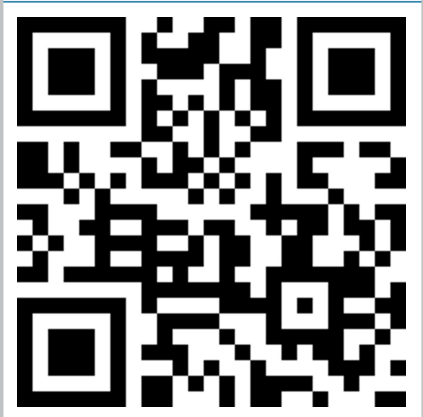

Point your SmartPhone at the code above. If you have a

QR code reader the video abstract will appear. Or use: http://dvpr.es//f8TCOB 
International Journal of Nanomedicine

Dovepress

\section{Publish your work in this journal}

The International Journal of Nanomedicine is an international, peerreviewed journal focusing on the application of nanotechnology in diagnostics, therapeutics, and drug delivery systems throughou the biomedical field. This journal is indexed on PubMed Central, MedLine, CAS, SciSearch ${ }^{\circledR}$, Current Contents ${ }^{\circledR} /$ Clinical Medicine,
Journal Citation Reports/Science Edition, EMBase, Scopus and the Elsevier Bibliographic databases. The manuscript management system is completely online and includes a very quick and fair peer-review system, which is all easy to use. Visit http://www.dovepress.com/ testimonials.php to read real quotes from published authors.

Submit your manuscript here: http://www.dovepress.com/international-journal-of-nanomedicine-journal 